\title{
Diffusion-Weighted Magnetic Resonance Imaging in Assessment of Primary Liver Cancer after HIFU Treatment
}

\author{
Sheng Li1, Sili Shi¹, Aiping Li2, Huihui Liu³ ${ }^{3}$ and Lili Cai ${ }^{4}$
}

\begin{abstract}
Objective: To determine the feasibility and role of diffusion-weighted magnetic resonance imaging (DWI) in the assessment of high-intensity focused ultrasound (HIFU) for the treatment of primary liver cancer.

Study Design: An observational study.

Place and Duration of Study: Department of Radiology, Renmin Hospital, Hubei University of Medicine, China, from July 2016 to March 2018.

Methodology: Forty-eight patients with primary liver cancer underwent conventional magnetic resonance plain scans, dynamic contrast-enhanced scans, and DWI before and two weeks after HIFU treatment, to compare and analyse the changes in apparent diffusion coefficient (ADC) and signal intensity (SI) in four regions of interest (the centre and the surrounding of the lesion, liver tissues around the lesion, back muscles) before and after HIFU treatment.

Results: Before HIFU treatment, the lesion showed high signals on the original DWI image, and the ADC image showed cool colours. Two weeks after HIFU treatment, the lesion showed low signals on the original DWI image, and ADC image showed warm colours; the ADC values of the centre and the surrounding of the lesion were significantly higher than those before treatment (both $p<0.001$ ); SI values of centre and surrounding of the lesion were significantly lower than those before treatment (both $p<0.001)$. One case $(2.08 \%$ ) each of residual tumor tissue and recurrence were observed and followed up for 3 months.

Conclusion: DWI can make a relatively accurate judgment on the therapeutic effect of HIFU for the treatment of primary liver cancer. Increased ADC values and reduced SI values can be used as markers for the evaluation of coagulative necrosis of primary liver cancer after HIFU treatment. In addition, DWI combined with dynamic contrast-enhanced MRI can make up for their own shortcomings.
\end{abstract}

Key Words: Magnetic resonance, Diffusion-weighted imaging, Primary liver cancer, High-intensity focused ultrasound, Therapeutic assessment.

\section{INTRODUCTION}

Primary liver cancer is the fifth most common cancer worldwide. Moreover, the number of deaths due to primary liver cancer is increasing markedly every year. Noninvasive and minimally invasive surgery for primary liver cancer is now at a high level platform with its rapid development. High-intensity focused ultrasound (HIFU) ablation is a new method for noninvasive and minimally invasive treatment of liver cancer. ${ }^{1,2}$ How to assess accurately the therapeutic effect of HIFU ablation in tumour treatment has become a research hotspot in recent years. Commonly used post-HIFU monitoring methods include CDFI, CEUS, and contrast-enhanced magnetic resonance imaging (CE-MRI), etc. ${ }^{3,4}$ Although CDFI and CEUS are convenient and quick examinations, their accuracy is relatively poor. CE-MRI is more accurate and can show the ablation of lesions in multiple

Department of Radiology 1 / Pain 2 / Nephrology 3 / Geratology 4 Renmin Hospital, Hubei University of Medicine, 442000,

China

Correspondence: Lili Cai, Department of Geratology,

Renmin Hospital, Hubei University of Medicine, 442000,

China

E-mail: kxenm3@163.com

Received: May 30, 2018; Accepted: October 30, 2018 sections. However, the time-consuming and expensive characteristics as well as the contraindications to MR, limit the clinical application of CE-MRI.5,6 In recent years, DWI has been gradually applied to the diagnosis, the differential diagnosis, and the therapeutic assessment of diseases in the liver and other organs. ${ }^{7} \mathrm{DWI}$ can reflect the water molecular movement in tumours and has potential value in tumour detection and tumour progression monitoring. ${ }^{8} \mathrm{DWI}$ can also avoid the use of contrast agents; and at the same time, can reflect the changes of the lesion before and after surgery at the molecular biological level, so as to accurately assess the effect of HIFU ablation. 9,10

The purpose of this study was to determine the feasibility and value of DWI in the therapeutic assessment of HIFU for the treatment of primary liver cancer.

\section{METHODOLOGY}

This study was conducted at the Department of Radiology, Renmin Hospital, Hubei University of Medicine, China, from July 2016 to March 2018. This Research was approved by the Hospital Ethical and Research Committee. A total of 48 patients with primary liver cancer were selected as the study subjects. Inclusion criteria were patients confirmed as primary liver cancer by biopsy or clinical diagnosis, and single 
lesion undergoing HIFU treatment. Exclusion criteria were patients who underwent interventional treatment or surgical treatment; who had intrahepatic or distant metastases; a history of contrast agent allergy; patients who were unable to cooperate with examinations due to severe liver, kidney and heart failure; other tumours.

For high-intensity focused ultrasound, patients' imaging data in terms of the location, size, shape of the lesion, and its relationship with the adjacent organs and blood vessels were obtained. The treatment range, working parameters, and the number of treatment slices were also designed. Then, under the colour Doppler ultrasound, localization and monitoring, an in vitro lineto-face conformal treatment was given with the treatment probe according to the slice order to treat tumours at each slice until the predetermined target area was completely covered.

Magnetic resonance examination was performed on all patients with primary liver cancer by using a 1.5T superconducting magnetic resonance imaging system and an 8-channel body phased array coil. Conventional magnetic resonance scans, dynamic contrast-enhanced $\mathrm{MRI}$, and DWI were performed on all patients before and two weeks after HIFU treatment. Dynamic contrastenhanced MRI scan parameters were dynamic enhanced three-dimensional volume acquisition (LAVA) scan of the liver with field of view of $32 \mathrm{~cm} \times 32 \mathrm{~cm}$, matrix of $256 \times$ 256, NSA=0.7. EPI sequences and frequency-selective fat suppression were used in DWI to remove chemical shift artifacts. The DWI data were transmitted to the GE AW 4.1 workstation, and the DWI images were postprocessed with Functool 2 software to obtain the pseudo-colour ADC images. The centre and the surrounding of the lesion, normal liver tissues around the lesion, and back muscles were set as regions of interest, to measure the ADC values and the signal intensity (SI). The area of ROI was taken as 55 60 $\mathrm{mm}^{2}$.

According to the characteristics of dynamic enhancement of lesions, tumor necrosis, survival or recurrence were judged. Tumor necrosis is defined as the appearance of no enhancement inside the lesion, appearance of regular annular enhancement around the lesion, or absence of regular annular enhancement on MRI dynamic enhancement after treatment. One week after treatment, MRI dynamic enhanced scanning showed irregular nodular, patchy or massive enhancement in arterial phase inside or / and around the lesion, which was defined as residual living tumor tissue or cells. The tumor recurrence was defined as a dynamic
MRI scan showing no enhancement inside the lesion within three months after treatment, but irregular nodular, patchy or massive enhancement around the lesion after three months.

The signal changes of the lesion in the DW and ADC images were analysed. The differences in the SI and $A D C$ values of three regions of interest (the centre of the lesion, the surrounding of the lesion, and the liver tissues around the lesion) before and two weeks after HIFU treatment were compared. The differences in the $\mathrm{SI}$ and ADC values of back muscles before and two weeks after HIFU treatment were compared.

SPSS version 21.0 statistical software package was used for data analysis. Measurement data were expressed as mean $\pm S D$. The paired t-test was used to compare the differences in the SI and ADC values of the centre of the lesion and liver tissues around the lesion before and after treatment. $P<0.05$ was considered statistically significant.

\section{RESULTS}

There were 30 males (62.50\%) and 18 females (37.50\%); aged 37-75 years, with an average age of $60.45 \pm 2.37$ years. Before HIFU treatment, the lesions of the 48 patients with primary liver cancer showed high signals on the original DW images, and cool colours on the ADC images. Two weeks after the HIFU treatment, the lesions of the 48 patients with primary liver cancer showed low signals on the DW images, and warm colours on the ADC images. After HIFU treatment, the patients were followed up for three months. One case $(2.08 \%)$ of dynamic enhancement showed uneven enhancement inside and around the lesion. Nodular high signal was seen on original DW images inside and around the lesion, ADC images were mixed with warm and cold colors, and MRI dynamic enhanced scan showed residual tumor tissue. One case $(2.08 \%)$ showed low signal and ADC showed warm color within two weeks after HIFU treatment. After three months of reexamination, irregular nodular high signals appeared around the lesion, ADC images showed cold color, and MRI dynamic enhanced scan showed recurrence of liver cancer.

Two weeks after HIFU treatment, the ADC values in the centre and the surrounding of the lesion were significantly higher than those before treatment (both $p<0.001$ ), while the ADC values of liver tissues around the lesion and back muscles were not significantly different from those before treatment $(p=0.433$ and 0.735 , respectively, Table I). The SI values in the centre

Table I: Changes in ADC values before and after HIFU treatment.

\begin{tabular}{|c|c|c|c|c|c|c|c|c|c|}
\hline \multirow[t]{2}{*}{ Time point } & \multirow[t]{2}{*}{$\mathrm{n}$} & \multicolumn{2}{|c|}{$\begin{array}{l}\text { ADC values in the } \\
\text { centre of the lesion } \\
\left(\times 10^{-3} \mathrm{~mm}^{2} / \mathrm{s}\right)\end{array}$} & \multicolumn{2}{|c|}{$\begin{array}{l}\text { ADC values in the } \\
\text { surrounding of the lesion } \\
\qquad\left(\times 10^{-3} \mathrm{~mm}^{2} / \mathrm{s}\right)\end{array}$} & \multicolumn{2}{|c|}{$\begin{array}{l}\text { ADC values in liver } \\
\text { tissues around the lesion } \\
\left(\times 10^{-3} \mathrm{~mm}^{2} / \mathrm{s}\right)\end{array}$} & \multicolumn{2}{|c|}{$\begin{array}{c}\text { ADC values of back } \\
\text { muscles } \\
\left(\times 10^{-3} \mathrm{~mm}^{2} / \mathrm{s}\right)\end{array}$} \\
\hline & & Mean \pm SD & p-value & Mean $\pm S D$ & $p$-value & Mean $\pm S D$ & $p$-value & Mean \pm SD & p-value \\
\hline Before HIFU treatment & 48 & $1.36 \pm 0.16$ & $<0.001$ & $1.39 \pm 0.19$ & $<0.001$ & $1.55 \pm 0.14$ & 0.433 & $1.78 \pm 0.17$ & 0.735 \\
\hline Two weeks after HIFU treatment & 48 & $1.95 \pm 0.41$ & & $1.82 \pm 0.36$ & & $1.54 \pm 0.17$ & & $1.77 \pm 0.24$ & \\
\hline
\end{tabular}


Table II: Changes in SI values before and after HIFU Treatment.

\begin{tabular}{|c|c|c|c|c|c|c|c|c|c|}
\hline \multirow[t]{2}{*}{ Time point } & \multirow[t]{2}{*}{$\mathrm{n}$} & \multicolumn{2}{|c|}{$\begin{array}{l}\text { SI values in the } \\
\text { centre of the lesion }\end{array}$} & \multicolumn{2}{|c|}{$\begin{array}{l}\text { SI values in the } \\
\text { surrounding of the lesion }\end{array}$} & \multicolumn{2}{|c|}{$\begin{array}{l}\text { SI values in liver } \\
\text { tissues around the lesion }\end{array}$} & \multicolumn{2}{|c|}{$\begin{array}{c}\text { SI values of back } \\
\text { muscles }\end{array}$} \\
\hline & & Mean \pm SD & $p$-value & Mean \pm SD & p-value & Mean $\pm S D$ & $\mathrm{p}$-value & Mean \pm SD & $\mathrm{p}$-value \\
\hline Before HIFU treatment & 48 & $194.15 \pm 49.12$ & $<0.001$ & $135.73 \pm 25.68$ & $<0.001$ & $95.66 \pm 4.11$ & 0.635 & $100.54 \pm 13.68$ & 0.911 \\
\hline Two weeks after HIFU treatment & 48 & $87.25 \pm 6.19$ & & $89.16 \pm 5.28$ & & $96.21 \pm 4.33$ & & $100.71 \pm 8.19$ & \\
\hline
\end{tabular}

and the surrounding of the lesion were significantly lower than those before treatment (both $p<0.001$ ), while the SI values of liver tissues around the lesion and back muscles were not significantly different from those before treatment $(p=0.635$ and 0.911 , respectively, Table II).

\section{DISCUSSION}

Primary liver cancer is a malignant cancer which has a high prevalence rate, and seriously threatens patients' lives. It is relatively insensitive to radiotherapy and chemotherapy, and leads to a short survival period.11 High-intensity focused ultrasound (HIFU) can kill tumour cells through instantaneous high temperature and cavitation in the focal region, and can block tumour blood supply. ${ }^{12,13}$ HIFU opens up a new noninvasive, safe and effective way to treat liver cancer. One study showed that HIFU offered a significant noninvasive therapy for local treatment of liver cancer; and increasing treatment time or repeated treatment may improve the efficiency of HIFU ablation treatment of right lobe liver cancers or with poor ultrasonic window. ${ }^{14}$

DWI technique is a research hotspot in recent years. The main principle is to use special sequences to record the Brownian motion of water molecules in vivo. ${ }^{15}$ This technique can reflect the microstructure of tissues by monitoring water molecules, and noninvasively detect the free motion of water molecules in body tissues. ADC is an important parameter in DWI, and is positively correlated with the degree of diffusion motion of water molecules. ${ }^{16,17}$ However, DWI signals are negatively correlated with the diffusion of water molecules. DWI can reflect the microstructure of tissues from the perspective of molecular biology without using contrast agents, and it only requires a few additional minutes of scanning after the conventional MR scanning. Therefore, the role of DWI in the diagnosis and therapeutic assessment of primary liver cancer has gradually been valued in clinical practice. ${ }^{18,19}$ Based on an accurate quantitative information of the free motion of water molecules provided by DWI, the pathological and physiological changes of primary liver cancer after HIFU ablation can be reflected indirectly. A relevant study confirmed that the ADC values of liver cysts and haemangiomas were significantly higher than those of primary liver cancer and metastatic tumours, with significant differences. ${ }^{20}$ The analysis of SI values may be a simpler and more direct method for therapeutic assessment. SI indicates the signal intensity of the lesion; the greater the value is, the stronger the lesion signal will be. This study found that two weeks after HIFU treatment, the ADC values of the centre and the surrounding of the lesion were significantly higher than those before treatment. The SI values of the centre and the surrounding of the lesion were significantly lower than those before treatment. The mechanism of increased $A D C$ and decreased SI in primary liver cancer after HIFU was probably because HIFU produced instantaneous high temperature, cavitation, and mechanical effects which caused coagulative necrosis of tumour cells. With increased membrane permeability, the cells ruptured and disintegrated; the cell membrane ruptured and lost its restriction to the intracellular water molecules. At the same time, the tumour cells dissolved and a large number of intracellular fluids leaked, causing the free diffusion of water molecules outside the cells and higher $A D C$ values.

Therefore, ADC can be used as an assessment index for primary liver cancer after HIFU treatment. This conclusion is basically consistent with previous reports. ${ }^{21}$ ADC combined with decreased DWI signals, an accurate judgement, can be made on the necrotic lesions after treatment. This method is more accurate, sensitive and specific. ${ }^{22}$ After HIFU treatment, the ADC values around the primary liver cancer increased and the $\mathrm{SI}$ values decreased, suggesting that the therapeutic range of HIFU was slightly larger than the lesion of primary liver cancer itself, and thus overcoming the risk of peripheral infiltration by the lesion.

This study also found that there were no significant changes in ADC and SI values in liver tissues around the lesion of primary liver cancer before and after HIFU treatment. It indicated that HIFU had an accurate location and no effects on the surrounding liver tissues. Thus, it is a safe, effective and noninvasive treatment.

In this study, the paraspinal muscles were used as the signal reference, and it was found that the ADC and SI values had no significant changes before and after HIFU treatment. It suggested that using DWI to assess the situations of primary liver cancer after HIFU treatment was feasible and repeatable. Moreover, the results of this study showed that after HIFU treatment, one case each of residual tumor tissue and of recurrence were followed up for three months. It is suggested that dynamic CE-MRI examination of the lesions after HIFU treatment can accurately determine the tumor necrosis, survival or recurrence. Therefore, DWI combined with dynamic CE-MRI can make up for their own shortcomings, provide more comprehensive information for 
clinical use, and better guide clinical selection of further treatment options.

However, due to some objective factors, there were still some deficiencies and shortcomings. Firstly, the deficiencies of this study lay in the inability to obtain patients' pathological data to confirm the results of the study. Secondly, due to the low spatial resolution of liver DW images, and the influences of respiratory motion and heartbeats, it was easy to produce a variety of artifacts, and could not reflect the details of the lesion. So, it cannot completely replace the conventional MR sequence. Thirdly, the time for judging HIFU efficacy review is short, and the long-term efficacy needs to be further studied in order to obtain more in-depth and detailed imaging features of patients with primary liver cancer after HIFU treatment.

\section{CONCLUSION}

DWI can make a relatively accurate judgment on the therapeutic effect of HIFU for the treatment of primary liver cancer. Increased ADC values and reduced SI values can be used as markers for the evaluation of coagulative necrosis of primary liver cancer after HIFU treatment. In addition, DWI combined with dynamic CE-MRI can make up for their own shortcomings.

\section{REFERENCES}

1. Wijlemans JW, Bartels LW, Deckers R, Ries M, Mali WPTM, Moonen CTW, et al. Magnetic resonance-guided high-intensity focused ultrasound (MR-HIFU) ablation of liver tumours. Cancer Imaging 2012; 12:387-94.

2. Williamson $\mathrm{T}$, Everitt $\mathrm{S}$, Chauhan $\mathrm{S}$. Automated geometric optimization for robotic HIFU treatment of liver tumors. Comput Biol Med 2018; 96:1-7.

3. Kim YS, Kim JH, Rhim H, Lim HK, Keserci B, Bae DS, et al. Volumetric MR-guided high-intensity focused ultrasound ablation with a one-layer strategy to treat large uterine fibroids: initial clinical outcomes. Radiology 2012; 263:600-9.

4. Lamby P, Prantl L, Fellner C, Geis S, Jung EM. Postoperative monitoring of tissue transfers: Advantages using contrast enhanced ultrasound (CEUS) and contrast enhanced MRI (ceMRI) with dynamic perfusion analysis? Clin Hemorheol Microcirc 2011; 48:105-17.

5. Zhang Y, Zhao J, Guo D, Zhong W, Ran L. Evaluation of shortterm response of high intensity focused ultrasound ablation for primary hepatic carcinoma: Utility of contrast-enhanced MRI and diffusion-weighted imaging. Eur J Radiol 2011; 79:347-52

6. Morana G, Alves CA, Tortora D, Severino M, Nozza P, Cama A, et al. Added value of diffusion-weighted imaging in pediatric central nervous system embryonal tumors surveillance. Oncotarget 2017; 8:60401-13.

7. Kenis C, Deckers F, Foer BD, Mieghem FV, Laere SV, Pouillon M. Diagnosis of liver metastases: Can diffusion-weighted imaging (DWI) be used as a stand alone sequence? Eur J Radiol 2012; 81:1016-23.
8. Shinya S, Sasaki T, Nakagawa Y, Guiquing Z, Yamamoto F, Yamashita $Y$. The usefulness of diffusion-weighted imaging (DWI) for the detection of gastric cancer. Hepatogastroenterology 2007; 54:1378-81.

9. Eiber M, Holzapfel K, Ganter C, Epple K, Metz S, Geinitz H, et al. Whole-body MRI including diffusion-weighted imaging (DWI) for patients with recurring prostate cancer: Technical feasibility and assessment of lesion conspicuity in DWI. $J$ Magn Reson Imaging 2011; 33:1160-70.

10. Li B, Cai W, Lv D, Guo X, Zhang J, Wang X, et al. Comparison of MRS and DWI in the diagnosis of prostate cancer based on sextant analysis. J Magn Reson e Imaging 2013; 37:194-200.

11. Akinyemiju T, Abera S, Ahmed M, Alam N, Alemayohu MA, Allen $C$, et al. The burden of primary liver cancer and underlying etiologies from 1990 to 2015 at the global, regional, and national level: Results from the global burden of disease study 2015. JAMA Oncol 2017; 3:1683-91.

12. Sengupta $S$, Balla VK. A review on the use of magnetic fields and ultrasound for non-invasive cancer treatment. J Adv Res 2018; 14:97-111.

13. Marinova M, Rauch M, Schild HH, Strunk HM. Novel noninvasive treatment with high-intensity focused ultrasound (HIFU). Ultraschall Med 2016; 37:46-55.

14. Chen L, Wang K, Chen Z, Meng Z, Chen H, Gao H, et al. High intensity focused ultrasound ablation for patients with inoperable liver cancer. Hepatogastroenterology 2015; 62:140-3.

15. Ben BD, Kronfeld-Duenias V, Zachor DA, Ekstein PM, Hendler T, Tarrasch $\mathrm{R}$, et al. Accelerated maturation of white matter in young children with autism: A high $B$ value DWI study. Neuroimage 2007; 37:40-7.

16. Choi HS, Kim AH, Ahn SS, Shin N, Kim J, Lee SK. Glioma grading capability: Comparisons among parameters from dynamic contrast-enhanced MRI and ADC value on DWI. Korean J Radiol 2013; 14:487-92.

17. Zhang $Y$, Huang $X$, Du H, Yun Z, Xin C, Peng W, et al. The value of diffusion-weighted imaging in assessing the $A D C$ changes of tissues adjacent to breast carcinoma. BMC Cancer 2009; 9:1-10.

18. Rosi G, Indino EL, Salvo V, Colarieti A, Fierro D, Scialpi M, et al. Role of multiparametric MRI in the diagnosis of prostate cancer: update. Urologia 2016; 83:61-7.

19. Sadinski M, Karczmar G, Peng Y, Wang S, Jiang Y, Medved M, et al. Pilot study of the use of hybrid multidimensional T2weighted imaging-DWI for the diagnosis of prostate cancer and evaluation of Gleason score. AJR Am J Roentgenol 2016; 207:592-8.

20. Tokgoz O, Unlu E, Unal I, Serifoglu I, Oz I, Aktas E, et al. Diagnostic value of diffusion-weighted $M R I$ and ADC in differential diagnosis of cavernous hemangioma of the liver. Afr Health Sci 2016; 16:227-33.

21. Yuan Z, Ye XD, Dong S, Xu LC, Sun ZC, Xiao XS. Water mobility of diffusion MRI in prediction of response to chemoembolization in liver cancer. Chin J Oncol 2009; 31: 293-7.

22. Feng J. Application of DWI combined with routine magnetic resonance imaging in follow-up of patients with primary liver cancer after treatment. Chin J CT MRI 2017; 15:100-2. 